\title{
A Review of the Application of Rate Theory to Simulate Vacancy Cluster Formation and Interstitial Defect Formation in Reactor Pressure Vessel Steel
}

\author{
Fallon Laliberte *, Lauren Boldon, and Li Liu \\ Rensselaer Polytechnic Institute: Mechanical Aerospace and Nuclear Engineering, $1108^{\text {th }}$ St., Troy, NY, USA
}

Received 31 August 2015; Accepted 19 October 2015

\begin{abstract}
The beltline region of the reactor pressure vessel (RPV) is subject to an extreme radiation, temperature, and pressure environment over several decades of operation; therefore it is necessary to understand the mechanisms through which radiation damage occurs and how it affects the mechanical and chemical properties of the RPV steel. Chemical rate theory is a mean field rate theory simulation model which applies chemistry to the evaluation of irradiation-induced embrittlement. It presents one method of analysis that may be coupled to other distinct methods, in order to analyze defect formation, ultimately providing useful information on strength, ductility, toughness and dimensional stability changes for effects such as embrittlement, reduction in ductility and toughness, void swelling, hardening, irradiation creep, stress corrosion cracking, etc. over time as materials are subjected to reactor operational irradiation. This paper serves as a brief review of rate theory fundamentals and presents several examples of research that exemplify the application and importance of rate theory in examining the effects of radiation damage on RPV steel.
\end{abstract}

Keywords: interstitial defect; vacancy formation; chemical rate theory; radiation damage; reactor pressure vessel steel.

\section{Introduction}

During the lifetime of a nuclear reactor, the reactor pressure vessel (RPV) experiences almost constant irradiation, particularly in the beltline region. As a result of this radiation bombardment, interstitials and vacancies aggregate in the crystal lattice and form extended defects, such as dislocation loops and voids. The dislocation loops and voids alter the macroscopic properties of the RPV steel and result in embrittlement and swelling. Radiation damage resistance can be improved by enhancing interstitial-vacancy recombination. Interstitial-vacancy recombination restores the crystal lattice and prevents these negative macroscopic mechanical changes in the material. Therefore, the formation of point defects and the recombination of interstitials and vacancies are competing processes.

Chemical rate theory may be utilized to describe the interplay between the formation of dislocation loops and voids and interstitial-vacancy recombination. This methodology incorporates the production rate of Frenkel pairs and interstitial-vacancy formation. The production rate of point defects is dependent on the amount of energy transferred to the lattice atoms by incident radiation (e.g. neutron, ion, electron). A review of the primary radiation damage formations of concern for RPVs-mainly displacement cascades-are presented in this paper. However, this paper is not meant to serve as a complete review of radiation damage theory, rather an outline of the primary modes of damage that are of concern with an emphasis on chemical rate theory.

\footnotetext{
* E-mail address: lalibf@rpi.edu ISSN: 1791-2377 @ 2015 Kavala Institute of Technology. All rights reserved.
}

Radiation damage effects on the structure and properties of materials became an increasingly popular area of research in the 1940s. In 1946, Wigner indicated the possibility of a damaging effect on material properties at high neutron fluxes, which was then later experimentally confirmed $[1,2,3]$. A decade later, Konobeevsky et al. discovered irradiation creep in fissile metallic uranium, which was then also observed in stainless steel [4,5]. During the 1950s and 1960 s great progress was made in the examination of crystalline defects from radiation exposure. It was recognized that atoms in solids migrate via vacancies under thermal equilibrium conditions and via vacancies and selfinterstitial atoms under irradiation [6]. In addition, during this time it was discovered that bombardment with energetic particles generates high concentrations of defects compared to equilibrium values, giving rise to radiation-enhanced diffusion [6].

By 1966, the interaction of various energetic particles with solid targets was understood very well, e.g. Kinchin and Pease model [7]. The primary radiation damage produced in materials was thought to consist of only Frenkel pairs, and it was believed that this damage would not result in serious long-term consequences in irradiated materials. This idea was correct to the extent that Frenkel pairs are mobile at operational temperatures common for irradiated reactor materials; self-interstitial atoms move and recombine, thereby restoring the crystal structure. For the most part, experiments confirmed this, and the defects did recombine. However, $1 \%$ of defects survived and formed vacancies, self-interstitial atom loops, and other defects [6]. Even though this is a minute fraction, the defects created in the irradiated material had a dramatic impact on their microstructure [8]. The discovery that these vacancies and self-interstitial atom loops greatly affect material behavior 
initiated a series of theoretical and experimental studies on the effects of radiation damage in reactor materials (such as RPV steel).

Shortly after the discovery of swelling in stainless steels, it was found that damage accumulation can occur with irradiation of any particle, given that the recoil energy is greater than the displacement threshold value $E_{d}$ (approximately 30 to $40 \mathrm{eV}$ for metals) [6]. Furthermore, it was determined that after irradiation, the microstructures of various materials were very similar, consisting of voids and dislocation loops. One crucial realization was that the microstructure developed under $1 \mathrm{MeV}$ electrons (which produced Frenkel pairs only) was similar to that formed under irradiation with fast neutrons or heavy ions, which generate more complicated primary radiation damage [9]. All of these developments resulted in an idea that threedimensional migrating point defects are the main mobile defect for any type of radiation. This assumption is the foundation of the initial kinetic models based on reaction rate theory (RT) [6]. The initial kinetic models are based on a mean-field approximation (MFA) of reaction kinetics with the production of only three-dimensional migrating Frenkel pairs [6]. The 3D migrating Frenkel pair models were developed in an attempt to explain the different phenomena observed in materials: radiation induced hardening, creep, swelling, radiation induced segregation and second phase precipitation. The methodology of this model is useful in the development of radiation damage theory, however it is valid for only $1 \mathrm{MeV}$ electron irradiation.

Further discoveries were made that contradicted the predictions made by the 3D migrating Frenkel pair modelsvoid super-lattice formation and the micro-scale regions of enhanced swelling near grain boundaries [10-14]. The void super-lattice formation and the micro-meter scale regions of swelling near grain boundaries proved that for neutron and heavy-ion radiation, the material microstructure evolves differently than what the 3D migrating Frenkel pair model had predicted. The 3D migrating Frenkel pair model views irradiation defect voids, dislocations, and second phase particles as being random [6]. Furthermore, the length scales available for the 3D Frenkel pair model do not correlate (they are an order of magnitude smaller) with the micrometer scale heterogeneities observed at the grain boundaries [6]. This indicated that the mechanisms of cascade damage for fast neutron and heavy ion irradiation differ from what the 3D migrating Frenkel pair model assumes.

Another model, the production bias model (PBM), arose in the 1990s by Woo and Singh [15-16]. The PBM model has evolved to explain high swelling rates at low dislocation density and grain boundaries and grain-size effects on void swelling and void lattice formation [17-27]. One advantage of PBM over the 3D migrating Frenkel pair model is the two features of the cascade damage: (1) the production of point defect clusters, in addition to single point defects, directly in displacement cascades, and (2) the 1D diffusion of single interstitial atom clusters, in combination with the $3 \mathrm{D}$ diffusion of point defects [6]. However, the PBM is limited in its application to low irradiation doses of less than 1dpa and the analysis of pure metals.

Despite its limitations at low doses, the PBM model can be applied at higher doses because it predicts a saturation of void size, due to the mixture of $1 \mathrm{D}$ and $3 \mathrm{D}$ diffusionreaction kinetics under cascade damage conditions $[18,28]$. Experimental work has shown unlimited void growth at high doses in most materials [29-32]. The PBM model fails to account for the indefinite void growth under cascade irradiation and explain the swelling saturation observed in void lattices [6]. Instead, the PBM predicts higher swelling rates in void lattices than in random void arrangements, due to the free channels between voids along close-packed crystal directions [24]. These "free channels" allow 1D migrating self-interstitial atoms an escape route to dislocations, thereby allowing $3 \mathrm{D}$ migrating vacancies to be stored in voids [32]. Figure 1 provides an overview of the time and size scales for which several of these methods are relevant and useful, in addition to the relevant phenomena scales. This article briefly reviews key concepts for radiation damage and chemical rate theory, and presents an overview of current research that has applied chemical rate theory to study neutron irradiation damage effects in RPV steel.

Phenomena

defect and solute

migration and clustering

single displacement multiple cascades, cascade cascade overlap

void swelling, hardening, embrittlement, creep,
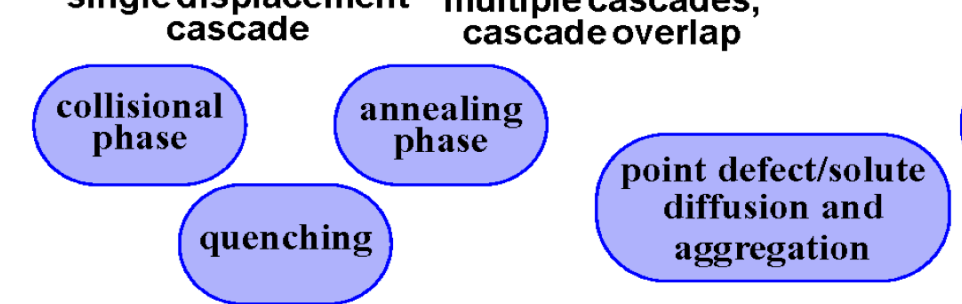
microstructure
evolution
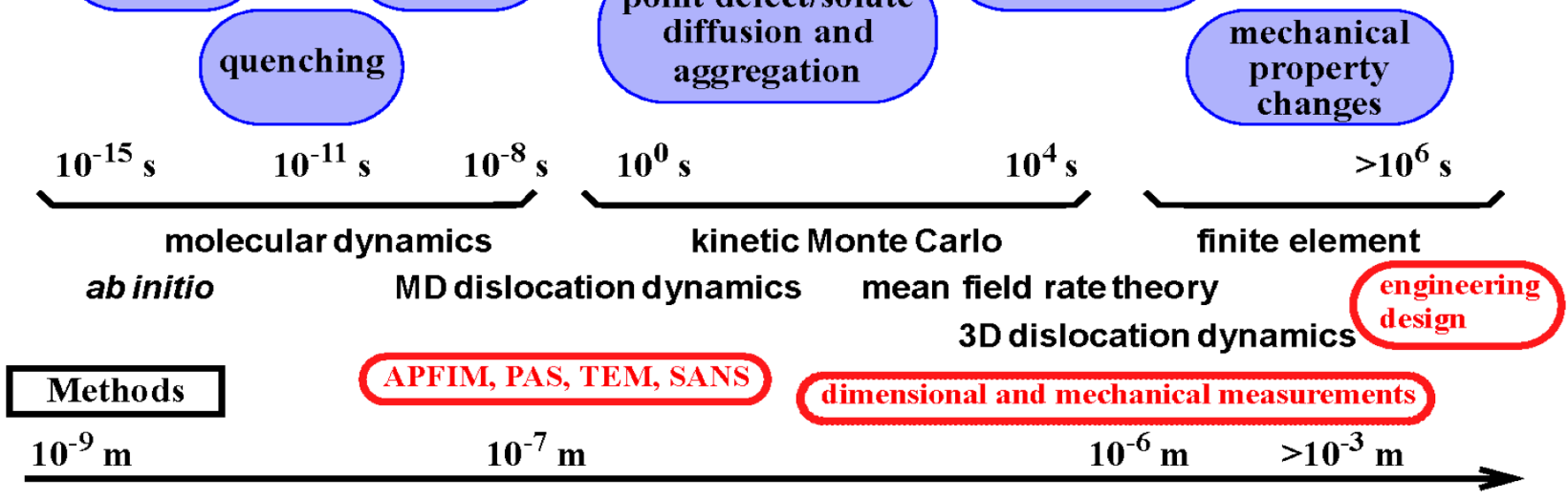

Fig. 1. Various radiation associated phenomena and methods to examine them. [1] 


\section{Radiation Damage and the Evolution of Rate Theory}

Atomic displacement results when a material is irradiated with energetic particles. Displacement cascades have temperature and energy as the primary statistical variations [1]. Secondary statistical variables are the microstructural length scales, surfaces, material structure (BCC, FCC, HCP), and any pre-existing radiation damage [1]. The cumulative effect of atomic displacement is the development of a preexisting microstructure and the formation of new defect structures. For example, radiation can induce Frank-faulted dislocation loops, second phase precipitates, cavity formation, and void formation in austenitic stainless steel [1]. Displacement cluster formation can also affect the mechanical properties of the metal like strain. Dense formation of defect microstructures at lower temperatures leads to increased hardening [1]. This phenomenon is particularly important for reactor pressure vessels (RPV). For example, the fracture toughness of an 800-ton RPV is severely degraded by radiation-induced defect structure with a size scale of $2-5 \mathrm{~nm}$ [1].

Fission reactions are one source of primary radiation damage in reactor systems. Fission fragments are heavy charged particles that recoil from fission events. They have a peak atomic mass of $90 \mathrm{amu}$ and $140 \mathrm{amu}$, an energy range of $80-100 \mathrm{MeV}$ and a limited range of travel [1]. Fission fragments primarily impact the fuel $\left(\right.$ e.g. $\left.\mathrm{UO}_{2}\right)$ so they will not cause significant damage to the RPV steel. High-energy neutrons (neutrons with energy greater than $1.0 \mathrm{MeV}$ ) and thermal neutrons (neutrons with energy less than $0.5 \mathrm{eV}$, $\mathrm{kT}_{\text {room }}=0.025 \mathrm{eV}$ ) generate displacements in the steel. Highenergy neutrons have a fission spectrum up to $20 \mathrm{MeV}$, with a peak at $0.65 \mathrm{MeV}$ [1] and a displacement cross-section minimum at $1 \mathrm{keV}$ (elastic scattering limit) for iron. Thermal neutrons produce low energy recoils from $(n, \gamma)$ capture reactions at the $100 \mathrm{eV}$ scale in steel [1].

High-energy electrons, with energies up to several $\mathrm{MeV}$, are produced by Compton scattering of fission gamma rays and cause displacement damage in the RPV steel. Similar to thermal neutrons, the high-energy electrons generate low energy recoils through elastic scattering [1]. Displacement from thermal electrons or neutrons can be significant to High Flux Isotope Reactor (HFIR), heavy water, or graphite moderated RPVs [1]. Lastly, a major source of radiation damage in RPV steels is nuclear transmutation products and solid products. For example, transmutation products of hydrogen and helium gas are produced from $(n, p)$ and $(n, \alpha)$ reactions, and solid products are produced via $(n, p)$ $(n, \alpha),(n, 2 n)$ and $(n, \gamma)$ reactions with subsequent $\beta$ decay [1]. The differences in neutron flux levels and energy spectrums result in distinct atomic displacement rates and primary knock-on atom (PKA) energy spectrums, respectively. The coolant alters the neutron energy spectrum, particularly at lower energies. High-energy neutrons, on the other hand, are influenced by the neutron source. The dpa cross section also exhibits energy dependence. The electronic stopping power is generally ignored for neutron irradiation of metals. The ratio of electronic to nuclear stopping power influences damage evolution in ceramics [1].

\section{Radiation Damage Theory}

Transition state theory (TST), or chemical reaction rate theory, is a method for calculating reaction rates. Radiation damage theory uses TST as a tool to describe reactions involving radiation produced defects, but cannot be reduced to it. This is true for the mean-field models and kinetic Monte Carlo (kMC) models that are also used to simulate radiation effects. Earlier models, such as the $3 \mathrm{D}$ migrating Frenkel Pair (FP3DM) model, failed to account for the discrepancy with experimental data, and therefore did not produce a significant contribution to the understanding of neutron irradiation effects on materials. Simulations that use the kMC model can be applied to obtain process information on a small time and length scale, however they cannot replace radiation damage theory for large-scale applications [6]. Radiation damage theory will continue to use reaction rate theory.

The mean field approximation is an important approximation for reaction rate theory. Mean field approximation (MFA), replaces all interactions for a manybody system with one interaction [6]. MFA is used in many areas of physics, for example, $a b$ initio and continuum models. For radiation damage, MFA is used to describe diffusion and interactions between defects in a selfconsistent way. Primary radiation damage for the MFA is generated by mobile vacancies, self-interstitial atoms, selfinterstitial atom clusters, and immobile defects [6]. Approximations such as this one are required, as microstructural evolution is too complex.

\subsection{Defect Productions and the Norgett-Robinson- \\ Torrens (NRT) Model}

When energetic particles interact with a solid target, they create primary knock-on (recoil) atoms (PKAs) by scattering with either incident radiation, e.g. electrons, neutrons, protons, or accelerated ions [6]. Some of the kinetic energy of the PKA, $\mathrm{E}_{\mathrm{PKA}}$, is transferred to electron excitation. The energy that is not transferred to electron excitation, $T_{d}$, is dissipated in elastic collisions between atoms. If $\mathrm{T}_{\mathrm{d}}$ exceeds the threshold displacement energy, $E_{d}$, for the target material, vacancy-interstitial pairs known as Frenkel pairs are generated. In the model proposed by Norgett et al. the total number of displaced atoms is proportional to the damage energy [33]:

$$
v(\tilde{E})=0.8 \frac{E^{P K A}(E)}{2 E_{d}}
$$

The total production rate of displacements per atom, dpa, symbolized at $\mathrm{G}^{\mathrm{NRT}}$, is calculated by integrating the flux of incident particles $\varphi(E)[33]$ :

$G^{N R T}=\int_{0}^{\infty} \mathrm{d} E \varphi(E) \int_{E_{d}}^{E^{\max }} \frac{d \sigma(E, E)}{d E} v(\tilde{E}) d \tilde{E}$

Note that $\sigma(E, \tilde{E})$ is the cross-section of reactions, in which an incident particle transfers energy $\tilde{E}$ to an atom, and $\tilde{E}^{\text {max }}$ is the maximum transferable energy [6]. The maximum transferable energy for a head on collision of a non-relativistic projectile of mass " $\mathrm{m}$ " and a target atom with mass " $M$ " is given by [6]:

$\tilde{E}^{\max }=\frac{4 M m}{(M+m)^{2}} E$

For relativistic electrons, the maximum transferable energy to an atom is given by [6]: 
$\tilde{E}^{\max }=\frac{2 m_{e}}{M}\left(\frac{E}{m_{e} c^{2}}\right) E$

Note that $m_{e}$ is the electron rest mass, and $c$ is the speed of light in $\mathrm{m} / \mathrm{s}$. This model - the NRT model — is accepted as an international standard for quantifying the number of atomic displacements produced under cascade damage conditions [6]. However, the NRT model cannot be used to characterize the defects formed during the collision phase and survive at the end of the cool-down phase of cascades. This is because the NRT model is based on isolated binary collisions.

\subsection{Characterization of Cascade-Produced Primary Damage}

Depending on the type of incident radiation and energy, one must use a model other than the NRT model to characterize cascade-generated primary radiation damage. The NRT displacement model is correct for $1 \mathrm{MeV}$ electrons, which produce only low-energy recoils, thus they generate Frenkel Pairs [6]. For higher recoil energies, the damage is produced in the form of displacement cascades. Displacement cascades change the production rate and the type of defects made. Molecular dynamics (MD) simulations have been used to investigate the cascade process [34, 35].

For incident radiation with energy greater than $0.5 \mathrm{keV}$, displacements are produced in cascades, which consist of collisions and cooling-down (recombining) [6]. A large fraction of defects are generated during the cooling-down stage of collision state of cascade recombination. The fraction of defects that did not recombine decreases with increasing PKA energy up to $10 \mathrm{keV}$ [6]. Around $10 \mathrm{keV}$ there is saturation at a value of approximately $30 \%$ of the NRT value [6]. At the end of the cooling stage, selfinterstitial atoms and vacancy clusters are formed. The fraction of defects in clusters increases with increasing PKA and is slightly greater for face-centered cubic (fcc) copper than bcc iron [6]. The self-interstitial atom clusters produced can be either glissile or sessile [6]. Glissile clusters with a large enough size-greater than 4 self-interstitial atoms in iron-migrate in 1D along close-packed crystallographic directions with a low-activation energy [36, 37]. The vacancy clusters created can be mobile or immobile vacancy loops, stacking-fault tetrahedral in fcc materials, or loosely correlated 3D arrays in bcc materials [36].

Cascade damage has several features: the generation rates of single vacancies and self -interstitial atoms are not equal and are less than what is predicted by the NRT model; the mobile species are 3D migrating single vacancies and self interstitial atoms, and 1D migrating self interstitial atoms and vacancy clusters; formation of sessile vacancy and self-interstitial atom clusters to serve as sources and sinks for mobile defects [6]. The point defect production rate is given by [6]:

$G_{v}=G^{N R T}\left(1-\varepsilon_{r}\right)\left(1-\varepsilon_{v}\right)$

$G_{i}=G^{N R T}\left(1-\varepsilon_{r}\right)\left(1-\varepsilon_{i}\right)$

Note that $G_{v}$ is the generation rate for vacancies, $G_{i}$ the generation rate for atom interstitials, and $G^{N R T}$ is the generation rate predicted by the NRT model, while $\varepsilon_{r}$ is the fraction of defects that recombined in cascades relative to the NRT standard, and $\varepsilon_{i}$ and $\varepsilon_{v}$ represent the fraction of self interstitial atoms and vacancies, respectively [6]. In addition, the model has parameters to describe mobile and immobile vacancy and self-interstitial (SIA) clusters of varying sizes. The production rate of the clusters containing $x$ defects, $G(x)$, depends on the cluster type and PKA energy and material, and is connected with the particular fraction of defects, $\varepsilon[6]$ :

$$
\sum_{x=2}^{\infty} x G_{\alpha}(x)=\varepsilon_{\alpha} G^{N R T}\left(1-\varepsilon_{r}\right)
$$

Note that $\alpha=v, i$ for the vacancy and SIA type clusters, respectively. The total fraction of defects in clusters is an additive relationship of the mobile and immobile clusters [6]:

$$
\varepsilon_{\alpha}=\varepsilon_{\alpha}^{s}+\varepsilon_{\alpha}^{g}
$$

The superscripts, $s$ and $g$, denote sessile and glissile clusters respectively. The mean size approximation defines the cluster production rate containing $x$ defects as [6],

$$
G_{\alpha}^{j}(x)=G_{\alpha}^{j} \delta\left(x-\left\langle x_{\alpha}^{j}\right\rangle\right)
$$

Where $j=s$ or $g$ for sessile and glissile clusters, respectively. Also note that $\delta(x)$ is the Kronecker delta, $\left\langle x_{\alpha}^{j}\right\rangle$ is the mean cluster size, and $G_{\alpha}^{j}$ is defined as [6],

$G_{\alpha}^{j}=\left\langle x_{\alpha}^{j}\right\rangle^{-1} G^{N R T}\left(1-\varepsilon_{r}\right) \varepsilon_{\alpha}^{j}$

This model assumes that small vacancy loops are not mobile and are sessile: $\varepsilon_{v}^{g}=0$ and $\varepsilon_{v}^{s}=\varepsilon_{v}$ [37].

\subsection{Point Defects}

Single interstitial atoms (SIAs) and interstitial atom clusters (SIA clusters) have been studied extensively since the 1930s because of their recognized effect on material properties. An understanding of SIAs and SIA clusters is critical to be able to describe the phenomena in solids under irradiation conditions. The properties and interactions of SIAs and SIA clusters differ significantly from vacancy type defects. As a result, the crystal behavior of the irradiated material differs from that under equilibrium conditions.

\subsubsection{Properties of Point Defects}

Both vacancies and SIAs are highly mobile at temperatures that are observed during reactor operation. The diffusion coefficient of SIAs, $D_{i}$, is greater than the diffusion coefficient for vacancies, $D_{v}$ [6]. This property leads to a specific temperature dependence of the damage accumulation, meaning that a limited number of defects can be accumulated at irradiation temperatures below the point when vacancies are immobile. As temperature increases beyond this limit, both vacancies and SIAs are mobile therefore defect accumulation is unlimited.

The relaxation volume of an SIA is much greater than for vacancy defects [6]. This results in higher interaction energy for SIAs with edge dislocations and additional defects. This property is the origin of the dislocation bias, and is the reason for void swelling in materials [38]. Furthermore, it is important to note that SIAs and vacancies are both defects of the opposite type whose interaction leads to mutual recombination. This property provides a decrease in the number of accumulated defects in the crystal during irradiation. Also, unlike vacancies, SIAs can exist in several different configurations, each possessing a different mechanism of migration [6]. Lastly, point defects-both vacancies and SIAs - are eliminated at fixed sinks, e.g. 
voids and dislocations [6]. This results in a variety of phenomena specific to point defects.

\subsubsection{Point Defect Clusters}

The configuration, thermal stability, and mobility of vacancy and SIA clusters are the factors that impact the kinetics of damage accumulation. These factors are different in fcc and bcc metals. In fcc metals, vacancy clusters are either dislocation loops or stacking fault tetrahedrals (SFTs). The configuration of vacancy clusters in fcc materials is dependent upon the stacking-fault energy and the fraction of clustered vacancies, $\varepsilon_{v}$, to that of SIAs, $\varepsilon_{i}$ [6]. In bcc metals, new vacancy clusters form $3 \mathrm{D}$ configurations and $\varepsilon_{v}$ is close to $\varepsilon_{i}$. In general, vacancy clusters are immobile and thermally unstable above the recovery stage temperature [6].

Unlike vacancy clusters, SIA clusters form 2D small dislocation loops. SIA clusters are thermally stable and highly mobile in 1D crystal directions [36]. Since SIA clusters can move in 1D before being trapped or absorbed by a vacancy, SIA clusters have distinct reaction kinetics compared to the 3D migrating defects and therefore have different damage accumulation than the Frenkel Pair 3D Migrating Model (FP3DM).

\subsection{Damage Accumulation}

One can separate defect types in crystals into two general categories: mobile defects (e.g. single vacancies, SIAs and vacancy clusters) and immobile defects (e.g. SIA loops and dislocations). The concentration of mobile defects is very small $\left(\sim 10^{-10}\right.$ to $10^{-6}$ per atom), while immobile defects can accumulate to several magnitudes greater in number. As a result, the mathematical description of mobile and immobile defects differs. Equations for mobile defects describe their reactions with immobile defects and are called rate equations. The description of immobile defects is more complicated due to nucleation, growth and coarsening effects [6].

Mobile defects produced by irradiation of the material are absorbed by immobile defects, e.g. voids and dislocation loops. According to the mean field approximation (MFA), a crystal can be treated as an absorbing medium. The absorption rate depends on the type of mobile defect, its concentration, and the size and spatial distribution of the immobile defects. The 'sink strength' parameter is introduced to describe the reaction cross-section or probability, $k_{v}^{2}, k_{i}^{2}$ and $k_{i c l}^{2}(x)$ for vacancies, SIAs, and SIA clusters where the number of SIAs in the cluster is denoted by " $x$ ", respectively [39, 40-43]. The following sections present a brief overview of the key equations necessary to study mobile and immobile defects and the resulting damage accumulation.

\subsubsection{Mobile Defects}

The following equations have several assumptions: point defects (PDs), single vacancies and SIAs migrate in 3D; SIA clusters are glissile and migrate in 1D; all vacancy clusters are immobile; the reactions between PDs and clusters are negligible; and immobile defects are distributed randomly over the volume [6]. For a concentration of mobile vacancies $C_{v}$, SIAs $C_{i}$, and SIA clusters $C_{i c l}^{g}(x)$, the balance equations are [6]:

$$
\begin{gathered}
\frac{d C_{v}}{d t}=G^{N R T}\left(1-\varepsilon_{r}\right)\left(1-\varepsilon_{v}\right)+G_{v}^{t h}-k_{v}^{2} D_{v} C_{v}- \\
\mu_{R} D_{i} C_{i} C_{v} \\
\frac{d C_{i}}{d t}=G^{N R T}\left(1-\varepsilon_{r}\right)\left(1-\varepsilon_{i}\right)-k_{i}^{2} D_{i} C_{i}-\mu_{R} D_{i} C_{i} C_{v}
\end{gathered}
$$

$$
\frac{d C_{i c l}^{g}(x)}{d t}=G_{i c l}^{g}(x)-k_{i c l}^{g}(x) D_{i c l} C_{i c l}^{g}
$$

Note, $x=2,3, \ldots, x_{\max }$ and $G_{v}^{t h}$ is the rate of thermal emission of vacancies from all immobile defects, e.g. dislocations and voids; $\mu_{R}$ is the recombination coefficient of point defects; and $D_{v}, D_{i}$, and $D_{i c l}(x)$ are the diffusion coefficients of vacancies, single SIAs, and SIA clusters, respectfully [6]. The mean size approximation can be applied, since the dependence of the cluster diffusivity $D_{i c l}(x)$ and the sink strength $k_{i c l}^{2}(x)$ on the size $x$ is weak $[36,37]$. The mean size approximation says that all defect clusters can be approximated to be of size $\left\langle x_{i}^{g}\right\rangle[36,37]$. Thus, Equation [13] can be reduced to [36, 37]:

$$
\frac{d c_{i c l}^{g}}{d t}=\left\langle x_{i}^{g}\right\rangle^{-1} G^{N R T}\left(1-\varepsilon_{r}\right) \varepsilon_{i}^{g}-k_{i c l}^{2}(x) D_{i c l} C_{i c l}^{g}
$$

Equation [10] is used to solve for the cluster generation rate. To solve Equations [11-14], one must know the sink strengths $\left(k_{v}^{2}, k_{i}^{2}, k_{i c l}^{2}\right)$, the rates of vacancy emission from various immobile defects to calculate the rate of thermal emission of vacancies from all immobile defects $\left(G_{v}^{t h}\right)$ and the recombination constant $\left(\mu_{R}\right)$.

\subsubsection{Immobile Defects}

Immobile defects are mainly formed during irradiation, and examples include voids, vacancy and SIA type dislocation loops and second phase precipitates. During irradiation, defects often nucleate, grow and coarsen, such that their size changes during irradiation. Description of immobile defects over time should include the size distribution function (SDF) $f(\xi, t)$, where $\xi$ is the cluster size. The measured SDF is represented in $\mathrm{x}$-space, $\xi=x$, where $x$ is the number of defects in a cluster: $f(x, t)$. The radius of a defect, $R$, is related to the number of point defects, $x$, it contains by [6]:

$$
\begin{aligned}
& \frac{4 \pi}{3} R^{3}=x \Omega \\
& \pi R^{2} b=x \Omega
\end{aligned}
$$

Note that Equation [15] and [16] describe the relationship between the radius of a defect, $\mathrm{R}$, and the number of point defects, $x$, for voids and loops, respectfully, where $\Omega$ is the atomic volume and $b$ is the loop Burgers vector [6]. The SDFs in R- and $\mathrm{x}$-space are related to each other by a simple relationship. If $d x$ and $d R$ are small and refer to the same cluster group, the number density of this cluster group is defined by the two functions and must be equal:

$f(x) d x=f(R) d R$

Equation [17] can be re-written as an equality of integrals for the total number density [6]:

$N=\sum_{x=2}^{\infty} f(x) \approx \int_{x=2}^{\infty} f(x) d x=\int_{R=R_{\min }}^{\infty} f(r) d R$

Therefore, the relationship between the radius of an immobile defect and the number of immobile point defects is: 


$$
f(R)=f(x) \frac{d x}{d R}
$$

Substituting Equations [15] and [16] into Equation [19], gives the following for voids and dislocation loops (the units of $f(x)$ are atom ${ }^{-1}$ or $\mathrm{m}^{-3}$, and of $f(R)$ is $\mathrm{m}^{-1} \times$ atom $^{-1}$ ):

$$
\begin{aligned}
& f_{v}(R)=\left(\frac{36 \pi}{\Omega}\right)^{\frac{1}{3}} x^{\frac{2}{3}} f_{v}(x)=\frac{4 \pi R^{3}}{3 \Omega} \\
& f_{v}(R)=\left(\frac{4 \pi b}{\Omega}\right)^{1 / 2} x^{1 / 2} f_{L}(x)=\frac{b \pi R^{2}}{\Omega}
\end{aligned}
$$

\subsubsection{Master Equation for Defects}

The master equation for the SDF, when the point defect cluster evolution is driven by the absorption of the point defects is [6]:

$$
\frac{\partial f^{s}(x, t)}{\partial t}=G^{s}(x)+\mathcal{F}(x-1, t)-\mathcal{F}(x, t), \text { for } x \geq 2
$$

Note that $G^{s}(x)$ is the rate of generation of the point defect clusters by an external source, e.g. by displacement cascades, and $\mathcal{F}(x, t)$ is the flux of the clusters in the sizespace. The flux, $\mathcal{F}(x, t)$ is defined as [6]:

$$
\mathcal{F}(x, t)=P(x, t) \times f(x, t)-Q(x+1, t) \times f(x+1, t)
$$

Note that $P(x, t)$ and $Q(x, t)$ are the rates of absorption and emission of point defects, respectively. The boundary conditions for the master equation illustrated in Equation [22] are the following, where $\mathrm{C}$ is the concentration:

$$
\begin{aligned}
& f(1)=C \\
& f(x \rightarrow \infty)=0
\end{aligned}
$$

If there are mobile point defects in the system, additional terms are added to the right-hand side of Equation [23] to account for interaction with immobile defects that can result in growth or decrease in the size-space [21]. The total rates of point defect absorption and emission are given by the following:

$$
\begin{aligned}
& \mathcal{F}_{\text {total, abs }}=\sum_{x=2}^{\infty} P(x) f(x) \\
& \mathcal{F}_{\text {total, emis }}=\sum_{x=2}^{\infty} Q(x) f(x)
\end{aligned}
$$

Note that Equations [26] and [27] are the total rates of point defect absorption and emission, respectively. $\mathcal{F}_{\text {total,abs }}$ and $\mathcal{F}_{\text {total,emis }}$ are related to the sink strength of the clusters, thereby serving as a link between equations for mobile and immobile defects. To provide an example, if voids with the SDF $f_{c}(x)$ and dislocations are only present in the crystal and the primary damage is in the form of Frenkel Pairs, the balance equations are given as [21]:

$$
\begin{aligned}
& \quad \frac{d C_{v}}{d t}=G^{N R T}\left(1-\varepsilon_{r}\right)-\left[\mu_{R} D_{i} C_{i} C_{v}+Z_{v}^{d} D_{v}\left(C_{v}-C_{v 0}\right)\right]- \\
& \left.\left[P_{c}(1) f_{c}(1, t)\right)-Q_{c}^{v}(2) f_{c}(2, t)\right]-\sum_{x=1}^{x=\infty}\left(P_{c}(x) f_{c}(x, t)-\right. \\
& \left.Q_{c}^{v}(x+1) f_{c}(x+1, t)\right) \\
& \quad \frac{d C_{i}}{d t}=G^{N R T}\left(1-\varepsilon_{r}\right)-\left[\mu_{R} D_{i} C_{i} C_{v}+Z_{i}^{d} \rho_{d} D_{i} C_{i}\right]- \\
& \sum_{x=1}^{x=0} Q_{c}^{i}(x+1) f_{c}(x+1, t)
\end{aligned}
$$

Methods to solve the master equation will not be discussed at length in this article. Briefly, the master equation is a continuity equation for the SDF of point defect clusters in a discreet space of their size. The master equation, Equation [22], provides one the most accurate descriptions of cluster evolution-under the mean-field approach describing all possible stages such as nucleation, growth and coarsening of clusters due to reactions with mobile defects [6]. Equation [22] is a set of coupled differential equations that describe the evolution of the clusters for a particular size(s). The master equation can be applied for a short period of time, i.e. a small number of cluster sizes, and solved numerically [44]. For a long period of time, to be able to accurately represent physical processes in the crystal lattice, the calculation must account for clusters containing a large number of point defects ( $\sim 10^{6}$ to $10^{12}$ atoms). Numerical integration of Equation [22] for such a system is possible, but requires extensive computational time.

An alternative to numerical integration is to solve Equation [22] via grouping techniques and differential equation approximations in a continuous space of sizes [45-56]. For example, the Fokker-Plank equation which approximates $P(x, t)$ and $Q(x, t)$ with continuous functions- $\tilde{P}(x, t)$ and $\tilde{Q}(x, t)$ - and replaces the right-hand side of Equations [22] and [23] by continuous functions of two variables: $\mathcal{F}(x, t)$ and $f(x, t)$ [6]. The Fokker-Plank equation is obtained from Equation [22] by expanding the right side in a Tailor series and excluding derivatives higher than the second order:

$$
\begin{gathered}
\frac{\partial f^{s}(x, t)}{\partial t}= \\
G^{s}(x)-\frac{\partial}{\partial x}[V(x, t) f(x, t)]+\frac{\partial^{2}}{\partial x^{2}}[D(x, t) f(x, t)],
\end{gathered}
$$

The first term, $G^{s}(x)$, describes the flow of point defect clusters, while the second term accounts for their diffusion in size-space. For large clusters, the evolution of the cluster is driven mainly by the first term, and the master equation and Fokker-Plank equations are equally accurate. For small clusters, the diffusion term dominates, therefore the FokkerPlank solution is not accurate and the master equation must be solved directly. The rates are defined as the following [6]:

$$
\begin{aligned}
& V(x, t)=\tilde{P}(x, t))-\tilde{Q}(x, t), \\
& \left.D(x)=\frac{1}{2}[\tilde{P}(x, t))-\tilde{Q}(x, t)\right],
\end{aligned}
$$

Note that nucleation typically occurs in the beginning of irradiation, therefore the defect clusters will be small and the diffusion term will dominate for the Fokker-Plank equation with small clusters. Thus, the Fokker-Plank equation will be less accurate than the master equation.

\subsection{Chemical Reaction Rate Theory}

Chemical reaction rate theory (CRRT) is an early model that is commonly referred to simply as rate theory, and is used to model damage accumulation under irradiation. CRRT has several assumptions. First, incident irradiation produces isolated FPs, single SIAs and vacancies in equal numbers; second, both SIAs and vacancies migrate in 3D; and third, the efficiencies of SIA and vacancy absorption by different sinks are distinct because of the respective strengths of the point defect-sink elastic collisions [57, 58]. Therefore, there is a preferential absorption of SIAs by dislocations. This 
dislocation bias is the driving force for the microstructural evolution of the CRRT model [57, 58].

The CRRT model is a variation of the FP3DM, discussed earlier. One major disadvantage of the FP3DM model is that various types of radiation are not distinguished; rather it is assumed in this model that initial radiation damage is in the form of Frenkel Pairs for all types of radiation. The CRRT model distinguishes between different types of radiation, and does not assume that initial radiation damage is always Frenkel Pairs. Recall that the FP3DM model is the simplest model, which accurately predicts the effects of $1 \mathrm{MeV}$ electron radiation damage.

\subsubsection{Reaction Kinetics of 3-D Migrating Defects}

For chemical rate theory, the equations for mobile defects are reduced to the following two equations [6]:

$$
\begin{aligned}
& \frac{d C_{v}}{d t}=G^{N R T}+G_{v}^{t h}-k_{v}^{2} D_{v} C_{v}-\mu_{R} D_{i} C_{i} C_{v}, \\
& \frac{d C_{i}}{d t}=G^{N R T}-k_{i}^{2} D_{i} C_{i}-\mu_{R} D_{i} C_{i} C_{v},
\end{aligned}
$$

To predict the evolution of mobile point defects and the impact of mobile defects on immobile defects, one must know the sink strength of different defects for vacancies and SIAs and the rate of their recombination [6]. The reaction kinetics of 3D migrating defects is a second order differential equation [39]. The leading term of the sink strength equation for chemical rate theory, for any individual defect, depends only on the characteristics of that defect [39]. The total sink strength, $k_{\alpha}^{2}$, is given as [6]:

$$
k_{\alpha}^{2}=\sum_{j=1}^{N} k_{\alpha j}^{2}
$$

Note that $\alpha=v, i$ for void and interstitial, respectfully, and $N$ is the total number of sinks per unit volume. The total sink strength of an ensemble of voids with the same radius $R$ is $k_{\alpha}^{2}=N k_{\alpha}^{2}(R)$. To find the individual sink strength of a void or dislocation loop, one must solve the point defect diffusion equation. To solve the point defect equation for individual sink strengths, one can apply the "Lossy-medium approximation" [42].

\subsubsection{Void Sink Strength Under Lossy-medium Approximation}

Given a system where mobile defects may undergo 3D diffusion near a spherical cavity of radius $R$, that is embedded in a Lossy-medium with sink strength, $k^{2}$ [6]:

$$
G-k^{2} D\left(C-{ }^{e q}\right)-\nabla \mathrm{J}=0,
$$

Note that $C^{e q}$ is the thermal-equilibrium concentration of mobile defects and the defect flux, $J$, is given by:

$$
J=-D\left(\nabla C+\frac{C}{k_{B} T} \nabla U\right)
$$

Note that $D$ is the diffusion coefficient, $U$ is the interaction energy of the defect with the void, $k_{B}$ is the Boltzmann constant, and $T$ is the absolute temperature. Boundary conditions for the defect concentration $C$ at the void surface and at infinity are:

$C(R)=C^{e q}$
$C(\infty)=C^{e q}+\frac{G}{k^{2} D}$

All other sinks in the system, voids and dislocations, are considered with the mean field approximation (MFA) and are included in the total sink strength, $k^{2}$. The solution to Equation [36] for a void located at the origin of the coordinate system, $r=0$, is [6]:

$$
C(r)=C^{e q}+\left(C^{\infty}+C^{e q}\right)\left(1-\frac{R}{r} e^{[-k(r-R)]}\right)
$$

The total defect flux, which is the flux through the void surface $S=4 \pi R^{2}$ is as follows [6]:

$$
I=-S(J(R))=k_{C}^{2}(R) D\left(C^{\infty}-C^{e q}\right),
$$

The void sink strength, $k_{C}^{2}(R)$, is defined as [6]:

$$
k_{C}^{2}(R)=4 \pi R(1+k R)
$$

To solve for the sink strength of all voids in the system, one must integrate over the SDF, $f(R)$ :

$$
\left.k_{C}^{2}=\int d R k_{C}^{2}(R) f(R)\right)=4 \pi\langle R\rangle N_{C}\left(1+k \frac{\left\langle R^{2}\right\rangle}{\langle R\rangle}\right)
$$

Note that $N_{C}=\int d R f(R)$ is the void number density, $\langle R\rangle$ is the void mean radius and $\left\langle R^{2}\right\rangle$ is the mean radius of the defect squared. A common value is $k^{2}=10^{14} \mathrm{~m}^{-2}$, hence one can omit the second term, leaving:

$$
k^{2}=4 \pi\langle R\rangle N_{C}
$$

The equations to describe sink strength of dislocations can be derived in the same fashion as that for voids. For further information regarding this and the sink strength of other defects, recombination, dissociation rate, and void growth rate in in 3D Reaction Kinetics model, the reader is referred to reference [6].

\section{Reactor Pressure Vessel Steels}

Light water reactor (LWR) pressure vessel steels are ferritic steels, which are designed to have high toughness and strength and to be weldable in thicker sections where the highest neutron dose is received (opposite the center of the reactor core) [59]. LWR reactor pressure vessel steels (RPV steels) tend to contain manganese, nickel and molybdenum. Pressurized water reactor (PWR) steel is more susceptible to embrittlement than boiling water reactor (BWR) steel, since BWRs experience less neutron irradiation [59]. PWR steels, therefore, are designed to handle higher neutron irradiation than are BWR steels. In addition to the radiation environment, the chemical composition of the steel contributes to the degree to which embrittlement occurs. Steels with a high proportion of copper and nickel are more susceptible to embrittlement, for example.

Age related degradation of the mechanical properties of RPV steels include: irradiation embrittlement, thermal ageing, temper embrittlement, fatigue and corrosion [59]. Neutron irradiation in RPV steels is a considerable factor in the aging process of the PRV. Table 1 lists the main ferritic materials used for LWR vessels and summarizes their chemical composition, while Table 2 displays the materials used in the "beltline" region of a LWR RPV [59]. 
Table 1. Chemical requirements - Main ferritic materials for reactor components in Western Countries [59]

\begin{tabular}{|c|c|c|c|c|c|c|c|c|c|c|c|c|c|c|}
\hline \multirow{2}{*}{ Designation } & \multicolumn{14}{|c|}{ Elements (mass \%) } \\
\hline & $\mathrm{c}$ & $\mathrm{si}$ & $\mathrm{Mn}$ & p & s & Cr & $\mathrm{Mo}_{0}$ & $\mathrm{Ni}$ & $\mathrm{v}$ & $\mathrm{cu}_{\mathrm{u}}$ & Al & $s_{n}$ & $N$ & $A_{s}$ \\
\hline ASTMA 3023 & 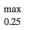 & 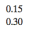 & 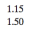 & $\frac{\max x}{0.035}$ & $\begin{array}{l}\max \\
0.0040\end{array}$ & & $\begin{array}{l}0.45 \\
0.60 \\
0.5\end{array}$ & & & & & & & \\
\hline ASTM A 336, Code Case 1236 & . 0.19 & 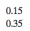 & $\begin{array}{l}1.10 \\
1.30\end{array}$ & $\max _{0.035}-x-y=$ & 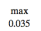 & $\max _{0.035}$ & $\begin{array}{l}0.50 \\
0.60\end{array}$ & 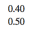 & & & & & & \\
\hline SMUE A SI8 C2 (1971) & 吕ax & 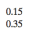 & $\begin{array}{l}0.00 \\
0.90 \\
0.90\end{array}$ & $\max _{0.025}$ & 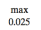 & $\begin{array}{l}0.25 \\
0.45\end{array}$ & $\begin{array}{l}0.55 \\
0.70 \\
0.70\end{array}$ & $\begin{array}{l}0.50 \\
0.90\end{array}$ & $\max _{0.05}^{\max }$ & & & & & \\
\hline SMEA S 533 OR B B(971) & $\begin{array}{l}\max \\
0.25\end{array}$ & $\begin{array}{l}0.15 \\
0.30 \\
0.5\end{array}$ & $\begin{array}{l}1.15 \\
1.50 \\
1\end{array}$ & $\max _{0.035}$ & $\max _{0.040}$ & & $\begin{array}{l}0.45 \\
0.650 \\
0.60\end{array}$ & $\begin{array}{l}0.40 \\
0.70\end{array}$ & & & & & & \\
\hline ASMEA A SIB C 2 (19985)" & 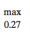 & $\begin{array}{l}0.15 \\
0.40 \\
0.40\end{array}$ & $\begin{array}{l}0.50 \\
1.00\end{array}$ & 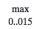 & 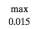 & .0.45 $0.25-3$ & $\begin{array}{l}0.55 \\
0.70 \\
0.5\end{array}$ & $\begin{array}{l}0.50 \\
1.00\end{array}$ & $\max _{0.05}$ & $\max _{0.15}$ & & & & \\
\hline ASMEA AIR C 3 (1989)" & $\begin{array}{l}\max \\
0.25\end{array}$ & $\begin{array}{l}0.15 \\
0.40 \\
0.45\end{array}$ & $\begin{array}{l}1.20 \\
1.50\end{array}$ & $\max _{0.015}$ & 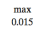 & 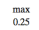 & 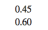 & $\begin{array}{l}0.40 \\
1.00\end{array}$ & $\max _{0.05}$ & & & & & \\
\hline ASME A S33Gr B (1989) & 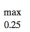 & $\begin{array}{l}0.15 \\
0.40 \\
0.03\end{array}$ & $\begin{array}{l}1.15 \\
1.50 \\
1.50\end{array}$ & $\max _{0.035}-x-y=$ & 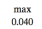 & & 迆 & $\begin{array}{l}0.40 \\
0.70\end{array}$ & & & & & & \\
\hline 16 MDDS RCC-M $2111^{b}$ & $\begin{array}{l}\max \\
0.22 \\
0\end{array}$ & 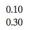 & 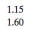 & $\max _{0.20}$ & 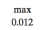 & $\begin{array}{l}\max \\
0.25\end{array}$ & $\begin{array}{l}0.43 \\
0.57\end{array}$ & $\begin{array}{l}0.50 \\
0.80 \\
0.02\end{array}$ & $\max _{0.01}^{\cos }$ & 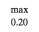 & $\begin{array}{c}\max \\
0.040\end{array}$ & & & \\
\hline 18 MDDS RCCM 2112 (1988) & 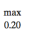 & 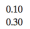 & $\begin{array}{l}1.15 \\
1.55\end{array}$ & $\max _{0.015}$ & 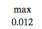 & $\underbrace{\max }_{0.25}$ & 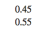 & $\begin{array}{l}0.50 \\
0.50\end{array}$ & $\max _{0.01}$ & 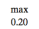 & $\underset{0.40}{\max }$ & & & \\
\hline 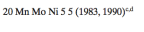 & . 0.17 & 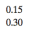 & $\begin{array}{l}1.20 \\
1.50\end{array}$ & $\frac{\max }{0.012}$ & 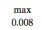 & $\max _{0.20}^{\max }$ & $\begin{array}{l}0.40 \\
0.55\end{array}$ & $\begin{array}{l}0.50 \\
0.50 \\
0.00\end{array}$ & $\max _{0.20}^{\max }$ & 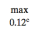 & $\begin{array}{l}0.010 \\
0.40 \\
0.40\end{array}$ & 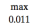 & $\underset{\substack{\max \\
0.013}}{ }$ & 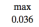 \\
\hline 22Ni Mo Cr 37 (1991) & $\begin{array}{l}0.17 \\
0.23 \\
0\end{array}$ & 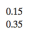 & $\begin{array}{l}0.50 \\
1.00\end{array}$ & $\max _{0.012}$ & 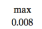 & $\begin{array}{l}0.25 \\
0.50\end{array}$ & 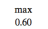 & $\begin{array}{l}0.000 \\
1.20^{\circ}\end{array}$ & $\max _{0.20}$ & 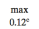 & $\begin{array}{l}0.010 \\
0.050\end{array}$ & 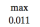 & 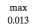 & $\underset{\substack{\max \\
0.036}}{x-3}$ \\
\hline
\end{tabular}

Table 2. Materials used for beltline region of LWR RPVs [59]

\begin{tabular}{|c|c|c|}
\hline Country & Shells & Austenitic cladding \\
\hline USA & $\begin{array}{l}\text { SA302 GR B } \\
\text { SA533 GR B, Class } 1 \\
\text { SA 508 Class } 2 \\
\text { SA 508 Class } 3\end{array}$ & $\begin{array}{l}\text { TYPE 308L, 309L } \\
\text { TYPE 304 }\end{array}$ \\
\hline France & $16 \mathrm{MnD} 5$ & \\
\hline Germany & $\begin{array}{l}\text { 20MnMoNi55 } \\
\text { 22NiMoCr37 }\end{array}$ & \\
\hline WWER-440 & 15Kh2MFA(A) & $\begin{array}{l}\text { Sv 07Kh25N13 - 1st layer } \\
\text { Sv 08Kh19N10G2B - 2nd layer }\end{array}$ \\
\hline WWER-1000 & $15 \mathrm{Kh} 2 \mathrm{NMFA}(\mathrm{A})$ & \\
\hline
\end{tabular}

The region of the RPV that is of primary concern regarding age-related degradation is the core beltline. The beltline of the RPV is the region of the shell material that directly surrounds the effective height of the fuel element assemblies, plus an additional volume of shell material below and above the reactor core [59]. The low alloy steels of the beltline are exposed to irradiation embrittlement that can lead to a loss of fracture toughness. The susceptibility of the RPV beltline steels to radiation-induced fracture has been shown to be strongly influenced by the presence of copper, nickel and phosphorous.

\section{Application of Rate Theory to Simulate Neutron Irradiation to Reactor Pressure Vessel Steel}

Chemical rate theory is a mean field rate theory simulation technique based upon chemistry that is applicable to the evaluation of radiation-induced embrittlement in reactor pressure vessel steels (RPV). A displacement cascade is created when neutrons interact with RPV steels; this cascade contains vacancy and interstitial clusters in addition to defects capable of migration, which will interact with the material through various mechanisms. The result is often point defect clusters and copper-rich precipitate formation. The following studies are significant in analyzing the effects of displacement cascades, such as embrittlement, which reduces the fracture toughness and increases the yield strength over the often-lengthy operational time frames for reactors.

\subsection{Displacement Damage Rate, Dose Rate, Flux, and Fluence Dependence}

To ensure safety of operation, it is a necessity to accurately depict and model the decline in fracture toughness and increased embrittlement under intermediate neutron flux levels and at high temperatures and pressures. Rate theory is one method of computational simulation that can address this issue and develop radiation-induced hardening models. Neutron flux and dose levels in the operational range (low to intermediate) are of particular concern, and data is not well determined [60]. Thus, illustrating the radiation effects becomes increasingly difficult. Stoller developed a kinetic model for embrittlement in order to assess these effects at relevant flux levels $\left(10^{12}-10^{15} \mathrm{n} / \mathrm{m}^{2} / \mathrm{s}\right.$, which result in $\left.10^{-9}-10^{-11} \mathrm{dpa} / \mathrm{s}\right) \quad[60]$ Typical surveillance experiments for embrittlement often result in 3-5 times greater flux than the maximum observed RPV flux; similarly material sensitivity to radiation is generally analyzed at 10 to 1000 times greater flux [60]. Although Stoller's model can help predict long-term radiation effects in a shorter period of time, its predictions may not directly correlate to long-term low flux levels. Stoller also states that the neutron flux data also differs in many variables, such as temperature, neutron energies, fluence, etc., which will all determine the applicability of experimental results to commercial reactors [60].

The kinetic embrittlement model utilizes rate theory in the analysis of point defects, clusters, and copper precipitate over time, where the mean lifetime for the displacement cascades was determined from the temperature-dependent vacancy evaporation rate and the instantaneous point defect fluxes [60]. The primary source term for damage was taken from prior molecular dynamics simulations [60]. Several conditions were assumed: precipitate nucleation was not incorporated; vacancy clusters operate mainly in recombination; vacancy mechanism was used to describe copper diffusion enhanced by radiation; diffusion kinetics and copper diffusion were used to detail precipitate development and growth; defect clusters were determined through a model for dislocation barrier hardening; and the hardening as a result of copper precipitate formation was determined from the Russell-Brown model [60]. All of these individual hardening contributions were then combined using the sum of the square roots for the shear strength to calculate the all-inclusive hardening effects [60]. This is directly related to the yield strength through simple application of the Taylor factor [60].

The kinetic model obtained simulation results that were similar to surveillance results in the RPV range for light water reactors and at a temperatures near $290^{\circ} \mathrm{C}$ [60]. Only a basic set of parameters were used, and through optimization of these parameters, the results may be improved. For this purpose, Stoller investigated the effects that many of his parameter assumptions had on the model [60]. For example, displacement rate impacts the model for RPV steel hardening through two prominent mechanisms: the first mainly influences defect clusters and results from defect formation and dissolution as unstable, competing processes; the second influences copper precipitation and results from the effect that displacement rate has on radiation-enhanced diffusion [60]. Thus, in low copper RPV steel, copper induced hardening is minimized when displacement rates are low, regardless of the dose, and cluster hardening is the primary method of hardening. On the other hand, at low displacement rates in high copper RPV steel, the fluence is less, which inhibits some hardening due to the presence of copper precipitates [60]. Embrittlement is not easy to predict in terms of damage rate dependence, and further study into copper content, fluence, irradiation temperature, etc. must be analyzed to improve the kinetic model.

Lee et al. utilized a similar method of rate theory analysis to determine yield strength changes that demonstrated reasonable agreement with surveillance data obtained for Korean LWRs [61]. In this analysis, the point 
defect cluster model followed that of Stoller, Kwon and Motta [62, 63]. The primary parameters included the concentrations of interstitial and vacancy atoms, the density of clusters containing vacancies and interstitial atoms of different sizes, and the copper precipitate sizes [61]. Orowan's model was then utilized to determine hardening as a result of the point defect clusters and copper precipitates [61].

Kwon et al. analyzed point defect clusters in stainless steel SS 304 at temperatures below $300^{\circ} \mathrm{C}$ to assess the effects on hardening [64]. In this model of rate theory, hardening was assumed to occur primarily through the interstitial and vacancy point defect behaviors; nucleation and growth mechanisms were achieved through diffusion reactions; MD simulation was used to determine point defect clustering as a result of displacement cascades; dislocation barrier model was used in hardening calculations; and a parametric study of several variables was performed as part of parameter optimization [64]. Kwon et al. developed a model that is applicable to other types of steels and alloys and can estimate hardening through an increase in yield strength. However, this model has uncertainties due to limitations in material and kinetic parameters and as a result of only incorporating point defect clusters [64].

Kwon et al. also modeled both point defect clusters and copper precipitates with reaction rate theory, in order to demonstrate how hardening is affected [65]. These results were then compared with surveillance data and postirradiation data obtained from French PWRs and Korean high-flux advanced neutron application reactor, respectively [65]. In comparison to the experimental data, Kwon et al. observed the inclusion of copper precipitates over predicted the amount of hardening and increase in yield strength in low-copper materials [65]. The data obtained from reaction rate theory was within $20 \%$ of that obtained from the surveillance data [65]. This may be a result of the kinetic and material parameters selected. In general, copper-rich precipitates play a significant role in radiation hardening, whereas point defect clusters are less effective according to the model. However, low-copper materials subjected to irradiation for a short time frame are primarily affected by point defect clusters, such that copper precipitate effects may be negligible [65]

Odette et al. reported an evaluation of dose rate effects, using a large database of irradiation hardening for RPV steels with a wide range of compositions. The RPV steels were irradiated at $290^{\circ} \mathrm{C}$ for three flux regimes, for a wide range of overlapping fluences [66]. Odette et al. found that the contribution of copper precipitates to irradiation hardening, increased to a plateau, which was a strong function of the $\mathrm{Cu}, \mathrm{Ni}$ and $\mathrm{Mn}$ content [66]. Furthermore, it was found that this plateau region was independent of the dose rate. The "pre-plateau" region shifted to a higher fluence with increasing dose rate. The irradiation hardening data was analyzed with a model that described: [I] the excess vacancy concentration under irradiation as a function of the dose rate (this included effects of solute vacancy traps for recombination); [II] radiation enhanced $\mathrm{Cu}$ diffusion coefficient; [III] the resulting accelerated growth of copper rich precipitates; and [IV] the contribution of copper rich precipitates to irradiation hardening [66]. Recombination of vacancies and self-interstitial atoms was shown to increase with a higher alloy $\mathrm{Ni}$ and $\mathrm{Mn}$ content. Despite of recombination rates, radiation enhanced diffusion demonstrated a high diffusion coefficient, much higher than predicted with simple rate theory [66]. The results of this study provide a method that can be applied to evaluate dose rate effects on $290^{\circ} \mathrm{C}$ hardening and embrittlement of RPV steels.

Watanabe et al. studied the displacement rate dependence of vacancy clusters and interstitial atom formation at various temperature conditions in alpha phase iron, which makes up a large proportion of low activation steels [67]. Defect cluster formation was analyzed numerically through mean field cluster dynamics simulation under several assumptions: free migration of vacancy and self-interstitial atoms occurs; Frenkel pairs resulting from irradiation are evenly dispersed in the steel; dislocation bias towards self-interstitial atoms is observed; thermal stability was addressed through size dependence of the cluster; and vacancy and self-interstitials may recombine to eliminate each other [67]. The observed rate theory results indicate that the nucleation behavior is distinct for clusters of vacancies and self-interstitial atoms, and the peak temperature of the nucleation rate for vacancy clusters is dependent on the displacement damage rate [67]. There is no temperature dependence observed for the self-interstitial atom clusters [67].

Flux dependence in RPV materials is extremely difficult to study, since the irradiation time necessary at lower fluxes becomes unwieldy; the fluence must be held constant; and the flux levels are correlated with the irradiation temperature, material composition, and the neutron energy spectrum [68]. However, the significance in understanding flux dependence is reflected in the use of surveillance studies for safety assessments, where high flux levels are typically used to predict material behavior in more reasonable time scales. Bergner et al. utilized rate theory modeling to explain the effect of copper on cluster formation observed by Small Angle Neutron Scattering (SANS) of RPV welds which were irradiated over a range of neutron fluxes and constant fluence and temperature [68]. A $0.22 \mathrm{wt}$. $\% \mathrm{Cu}$ weld was primarily investigated at fluence and temperature of $2.2 * 10^{19} \mathrm{~cm}^{-2}$ and approximately $285{ }^{\circ} \mathrm{C}$. The flux was varied from $6 * 10^{10}$ to $2.1 * 10^{12} \mathrm{~cm}^{-2} \mathrm{~s}^{-1}$, requiring irradiation times of 11.6 years and 122 days, respectively [68]. A weld containing only $0.03 \mathrm{wt} . \% \mathrm{Cu}$ was also investigated for comparison and to demonstrate how the presence of $\mathrm{Cu}$ dramatically influences cluster formation. The rate theory model was developed to better understand if copper was responsible for the observed flux effects. Bergner et al. explained the influence of flux on cluster growth in terms of two regimes - one at lower fluxes, where cluster growth is flux-independent, and one where cluster growth is affected by irradiation-enhanced copper diffusion, which is related to the flux level.

\subsection{Grain Boundary Defect Migration}

Ebihara et al. studied the grain-boundary segregation of phosphorous in A533B RPV steels as a result of carbon trapping sites under irradiation (Ebihara et al 2010). Rate theory was applied for comparison with Auger electron microprobe analysis of experimental results. In the rate theory model developed by Ebihara, the carbon atoms were treated as sites to trap both vacancies and self-interstitials. In this simulation, phosphorous, vacancies, and interstitials progress via migration, association, or dissociation, which are addressed through the diffusion rate equation. The carbon atoms were incorporated into the analysis through the first-principles calculation, such that migration does not occur as a result of irradiation; instead carbon atoms are dispersed evenly throughout the material and serve to trap 
vacancies or interstitials (Domain 2006; Ebihara et al.) Since the carbon atoms do not migrate, they influence the defects by slowing their progress through the material. The binding states between the carbon atoms and the vacancy or interstitial defects are incorporated directly into the diffusion rate equation. The Domain and Becquart four-frequency model for bcc structures and the Meslin et al. interstitial dumbbell model for phosphorous migration were assumed (Ebihara et al., Domain and Becquart; Meslin et al.).

The A533B steel was neutron irradiated at $290{ }^{\circ} \mathrm{C}$ under a fluence and dose rate of $6.3 \times 10^{19} \mathrm{n} / \mathrm{cm}^{2}$ and $1.7 \times 10^{-10} \mathrm{dps} / \mathrm{s}$, respectively. The Auger electron microprobe determined the phospohorous coverage prior to irradiation as 0.032 at wt.\%. All of these values were necessary for the simulation, which found a direct relationship between the phosphorous coverage at the grain boundary and the dose. At low dose regions, the phosphorous coverage increased substantially with an increase in dose. This effect leveled off to a slight increase at moderate to high dose regions. This trend followed the experimental data obtained closely, and was observed in phosphorous segregation both with and without the presence of carbon atoms. It was observed that carbon merely influences the diffusion speed of vacancy and interstitial defects which provide the mechanism for phosphorous migration to the grain boundary, so the same trend is observed, but at a lower phosphorous coverage as the presence of carbon atoms is increased.

Both the interstitial dumbbell model and the vacancy model for phosphorous migration were analyzed without the presence of carbon to ensure the validity of assumptions. It was determined that phosphorous primarily migrates to the grain boundary via the interstitial dumbbell model transport rather than the vacancy model. However, both of these models independently produced diffusion rate theory results for phosphorous coverage at the grain boundary, which was representative of the experimental data. Similarly, the effect of carbon atoms on vacancies and interstitials were also evaluated, and it was determined that carbon atoms influence primarily vacancy diffusion and have minimal influence over the self-interstitial diffusion. All of the simulation data is dependent upon the sink strength selected for the vacancy and interstitial atoms; in this particular study, the phosphorus migrations occurred via interstitial dumbbell transport, but was also influenced by vacancy transport, as interstitials were reduced. Carbon atoms were found to affect the migration of phosphorous by reducing the availability of vacancies, as they slowed vacancy diffusion. This study is significant, as it demonstrates the mode of transport for phosphorous to the grain boundary, which results in embrittlement and a reduction in toughness. The formation of vacancy and interstitial clusters due to irradiation will influence the simulation data and must be further studied.

\subsection{Mobile Point Defect and Cluster Formation,} Evolution, and Dynamics

Birkenheuer et al. developed the Vacancy-Coupled Clustering (V3C) model as part of the PERFECT multi-scale project, incorporating rate theory models to explain the experimental results observed through Small-angle Neutron Scattering (SANS) [70]. The defect cluster volume fraction and the size distribution function peak radius for two iron alloys containing $0.3 \%$ and $0.1 \%$ copper, respectively, were assessed under a neutron flux of $0.95 * 10^{18} \mathrm{n} /\left(\mathrm{m}^{2} * \mathrm{~s}\right)$, temperature of $300{ }^{\circ} \mathrm{C}$, and various displacement damage doses [70]. The V3C model only looked at copper-rich precipitate absorption of vacancies; pure copper precipitates were not included. This model compared well with the SANS results, once the thermal diffusivity and interface energies for the two iron alloys were taken into account [70].

\subsection{Thermodynamic Effects of Defect Formation}

Straalsund evaluated neutron irradiation-induced void formation and its temperature dependence, and determined that the minimum temperature for void formation using cluster dissolution kinetic theory may be lower than prior models estimated-and much closer to experimentally observed data [71]. Void growth is dependent on the nucleation rate, temperature, helium effects, displacement damage, etc. Simple void growth theory implies that a temperature shift is observed to equate low and high rates of displacement damage [71]. Typically, free combination is the only effect incorporated in the temperature shift calculations. For the vacancies which do not recombine within a displacement collision and are present as vacancy clusters, Straalsund proposed the cluster dissolution rate be utilized rather than the migration rate as the limiting condition [71]. This resulted in lower values for temperature shifts below the maximum swelling vs. temperature curve, and is more representative of the displacement dependence and the defects surviving annihilation in the cascade [71].

Al-Motasem et al. studied the temperature behavior of nano-sized clusters containing vacancies and/or copper in polycrystalline bcc iron with rate theory and atomistic simulations. The initial configuration and energetics were obtained from Monte Carlo and molecular dynamics simulation, while the phonon influence on the clusters was determined through the dynamical matrix method [72]. The results indicated the total free binding energy for clusters containing only vacancies decreased at $0 \mathrm{~K}$, while the binding energy for clusters containing only copper increased at the same temperature. The behavior for clusters containing both copper and vacancies was inconsistent.

\subsection{Helium and Hydrogen Microstructural Effects}

Reduced-activation-ferritic/martensitic (RAFM) steels are being investigated for their potential use in fusion reactors as the blanket material, and as a result may be subjected to very high energy neutron irradiation (14 MeV) [73]. Such high energy neutrons induce many types of point defects. Watanabe et al. used mean field cluster modeling (reaction rate theory) to the study how helium impacts the evolution of interstitial dislocation loops and bubbles in RAFM steels at $723 \mathrm{~K}$ with Frenkel pair and helium production rates of $10^{-6} \mathrm{dpa} / \mathrm{sec}$ and $0,10^{-7}$, or $10^{-5} \mathrm{appm} \mathrm{He} / \mathrm{sec}$ [73]. In this analysis, lath martensite $(\mathrm{Fe}-8 \mathrm{Cr})$ was assumed to contain a uniform distribution of dislocations and precipitates as well as constant dislocation density [73]. The Frenkel pairs and helium atoms were uniformly created as a result of atomic displacement and nuclear transmutation, respectively [73]. Recombination was considered between vacancies and self-interstitials, while helium was only considered to react with vacancies to form bubbles (no interstitial interactions [73]. Finally, the helium bubbles and I-loops were considered spherical and discoidal, respectively [73]. Watanabe et al. concluded that the presence of helium has a substantially different effect for I-loop and bubble formation, where I-loops exhibit nearly helium-independent behavior, while bubble formation exhibits highly heliumdependent behavior. 
Magnesium oxide is significant in nuclear fuels and may be incorporated to improve thermal conductivity and performance of the fuel [75]. In serving this function, Magnesium oxide will have to undergo neutron irradiation and helium interactions, as helium is created during alpha decay within the fuel [75]. Runevall and Sandberg studied helium bubble formation with rate theory to determine how helium migrates and becomes trapped in magnesium oxide vacancy defects and clusters [75]. Several helium concentrations spanning temperatures from $300 \mathrm{~K}$ to $1600 \mathrm{~K}$ and a range of vacancy clusters were compared to positron annihilation Doppler broadening experimentation of magnesium oxide containing helium $[75,76]$. In this rate theory analysis, the trapping of helium in grain boundaries or dislocations was not considered; vacancies in oxygen and magnesium were considered immobile; helium interstitials migrated until captured in another cluster; and the magnesium oxide, magnesium-helium and helium-helium, and helium-oxygen interaction potentials were taken or derived from Grimes, Busker et al., and Grimes and Catlow, respectively $[75,77,78,79)$. Runevall and Sandberg observed good correlation with the helium annealing experimental data. They also determined that helium acted as stabilizing force on larger vacancy clusters, but void and bubble growth was highly dependent upon both the temperature and concentration of helium present (75).

Hydrogen and helium material interactions will alter the mechanical properties of structural materials, such as RPV steels. Although hydrogen retention in materials is related to the number and concentration of trapping sites, the mechanisms with which it is captured and retained is still widely unknown. Tostolutskaya et al. analyzed the displacement damage effects on the following ferriticmartensitic steels due to helium, hydrogen, and argon ions: EP-450, EP-852, and RUSFER-EK-181 [80]. In this study, ion implantation, nuclear reaction depth profiling, and thermal desorption spectrometry mechanisms were utilized to assess ion retention and detrapping, specifically for deuterium, while continuum rate theory provided the simulation necessary to determine relevant thermodynamic properties for the deuterium processes [80]. The results demonstrated that in the portions of steel containing only defects (no helium or argon atoms), hydrogen retention was attributed to vacancies, while in the portions exhibiting both displacement damage defects and inert helium or argon atoms, trapping occurred at higher levels due to bubble formation and stress fields [80]. The effect of gas bubble impurities on hydrogen trapping was significantly more prominent than that of displacement damage effects, demonstrating how the implantation of noble gases results in steels with higher hydrogen trapping and retention capabilities [80].

\section{Conclusions}

Radiation damage is an important subject to study because the strength, ductility, toughness and dimensional stability are determined by defect structure. For example, the grain size, dislocation density, and size and density of second phase precipitates are impacted by the defect structure. Development of new materials to better withstand the harsh environments of a nuclear reactor is dependent on the understanding of how these materials will react under intermediate to high flux radiation at high temperatures and pressures. Radiation damage simulation therefore plays a crucial role in reactor material design.

Chemical rate theory is one method of analysis that may be used in analyzing the radiation damage in the reactor pressure vessel. Although rate theory details information on damage accumulation without discriminating on defect type, there are several limitations in its use for RPV analysis. These include the dependence on the assumed flux, fluence, irradiation temperature, damage displacement rate, dose, etc. in addition to the material properties present, such as the fraction of copper and other diffusing species (hydrogen, helium, carbon, nitrogen, etc.); the accuracy of microstructural and kinetic data, such as the formation and migration energies for point defects and interstitial atoms; and the computational time necessary for higher displacement damage and dose rates. This article presented relevant background theory on radiation damage, a review of fundamental chemical rate theory, an overview of the materials and radiation damage effects of the reactor pressure vessel, and the potential integration of chemical rate theory applications in this field of study. Moving forward, it is likely that rate theory must be coupled with other techniques to understand the full array of irradiation effects on materials and to overcome the limitations of the individual assessment techniques.

\section{Acknowledgments}

This material is based upon work supported under a Department of Energy Nuclear Energy University Programs Graduate Fellowship. Any opinions, findings, conclusions or recommendations expressed in this publication are those of the author(s) and do not necessarily reflect the view of the Department of Energy Office of Nuclear Energy.

\section{References}

1. Stoller, Roger E. Radiation Damage: Mechanisms and Modeling. Joint EFRC Summer School Presentation. (11 May 2015) http://cdp.ornl.gov/EFRCschool/pdf/talks/Stoller.pdf.

2. Wigner, E.P. Theoretical Physics in the Metallurgical Laboratory of Chicago. Journal of Applied Physics. 1946, Volume 17, Issue 11, Pages: 857-863.

3. Seitz, F. Radiation Effects in Solids. Physics Today. 1952, 5(6), 69.

4. Konobeevsky, S.T.; Pravdyuk, N.F.; Kitaitsev, V.I. Effect of irradiation on structure and properties of fissionable materials. In Proceedings of International Conference on Peaceful Uses of Atomic Energy United Nation, New York; 1955; Vol. 7, pp. 433440.

5. Joseph, J.W., Jr. Stress Relaxation in Stainless Steel During Irradiation; USAEC Report DP-369; E.I. Dupont de Nemours and Co., 1955.
6. Golubov, S.I., Radiation Damage Theory. Comprehensive nuclear materials. Volume 1, Basic Aspects of Radiation Effects in Solids/Basic Aspects of Multi-Scale Modeling. Pp. 357-391. 2012.

7. Kinchin, G.H; Pease, R. S. The displacement of atoms in solids by radiation. Rep. Prog. Phys. 1955, 18, 1-51.

8. Cawthorne, C.; Fulton, E.J. Voids in irradiated stainless steel. Nature 1967, 216, 575-576.

9. Singh, B.N.; Trinkaus, H.; Golubov, S.I. In Encyclopedia of Materials: Science and Technology; Buschow, K.H.J., Cahn, R.W., Flemings, M.C., Ilschner, B., Kramer, E.J., Mahajan, S., Eds.; Oxford: Pergamon, 2001; Vol. 8, pp 7957-7972.

10. Evans, J. H. Observations of a regular void array in high purity molybdenum irradiated with $2 \mathrm{MeV}$ nitrogen atoms. Nature $\mathbf{1 9 7 1}$ $229,403-404$. 
11. Evans, J. H. Observations of a regular void array in high purity molybdenum at T.Z.M. irradiated at high temperatures with 2 $\mathrm{MeV}$ nitrogen ions. Radiat. Eff. 1971, 10, 55-60.

12. Kulchinski, G. L.; Brimhall, J. L.; Kissinger, H. I. J. Nucl. Mater. 1971, 40, 166-174.

13. Wiffen, F. W. In Proceedings of the 1971 International Conference on Radiation-Induced Voids in Metals (CONF 710601); Corbett, J. W., Ianniello, L., Eds; U.S. Atomic Energy Albany: New York, 1972; pp 386-396.

14. Farrell, K.; Houston, J. T.; Wolfenden, A.; King, R. T.; Jostsons, A. In Proceedings of the 1971 International Conference on Radiation Induced Voids in Metals (CONF 710601); Corbett, J. W., Ianniello, L., Eds.: U.S. Atomic Energy Albany: New Yor, 1972; pp 376-385.

15. Woo, C. H.; Singh, B. N. The Concept of Production Bias and Its Possible Role in Defect Accumulation under Cascade Damage Conditions. Phys. Status Solidi B 1990, 159, 609-616.

16. Woo, C. H.; Singh, B. N. Production Bias due to Clustering of Point Defects in Irradiation-induced Cascades. Philos. Mag. A 1992, 65, 889-912.

17. Singh, B. N.; Foreman, A. J. E. Production bias and void swelling in the transient regime under cascade damage conditions. Philos. Mag. A 1992, 66, 975-990.

18. Trinkaus, J.; Singh, B. N.; Foreman, A. J. E. J. Nucl. Mater. 1992, 199, $1-5$.

19. Trinkaus, H.; Singh, B. N.; Foreman, A. J. E. J. Nucl. Mater. 1993, 206, 200-211.

20. Trinkaus, H.; Singh, B. N.; Woo, C. H. J. Nucl. Mater.1994, $212-$ $215,168-174$.

21. Singh, B. N.; Golubov, S. I.; Trinkaus, H.; Serra, A.; Osetsky, Yu. N.; Barashev, A. V. J. Nucl. Mater. 1997, 251, 107-122.

22. Golubov, S. I.; Singh, B. N.; Trinkaus, H. Defect accumulation in fee and bcc metals and alloys under cascade damage conditionsTowards a generalization of the production bias model. J. Nucl. Mater. 2000, 276, 78-89.

23. Golubov, S. I.; Singh, B. N.; Trinkaus, J. O. N. Philos. Mag. A 2001, 81, 2533-2552.

24. Barashev, A. V.; Golubov, S. I.; Trinkaus, H. Reaction kinetics of glissile interstitial clusters in a crystal containing voids and dislocations. Philos. Mag. A 2001, 81(10), 2515-2532.

25. Singh, B. N.; Eldrup, M.; Zinkle, S. J.; Golubov, S. I. Philos. Mag. A 2002, 82, 1137-1159.

26. Trinkaus, H.; Heinisch, J. L.; Barashev, A. V.; Golubov, S. I.; Singh, B. N. Phys. Rev. B 2002, 66, 060105(R), 1-4.

27. Singh, B. N.; Golubov, S. I.; Trinkaus, J. A general treatment of one to three-dimensional diffusion reaction kinetics of interstitial clusters: Implications for the evolution of voids; Riso Report No. Riso-R-1622 (EN); 2008.

28. Barashev, A. V.; Golubov, S. I. J. Nucl. Mater. 2009, 389, 407409.

29. Singh, B. N.; Horsewell, A.; Gelles, D. S.; Garner, F.A. J. Nucl. Mater. 1984, 191-194, 1172-1176.

30. Garner, F. A. Evolution of microstructure in face-centered cubic metals during irradiation. J. Nucl. Mater. 1993, 205, 98-117.

31. Garner, F. A.; Toloczko, M. B.; Sencer, B.H. Behavior of fecaustenitic and bcc-ferritic/martensitic alloys at high neutron exposure. J. Nucl. Mater. 2000, 276, 123-142.

32. Matsui, J.; Gelles, D. S.; Kohno, Y. In Effects of Radiation on Materials: $15^{\text {th }}$ International Symposium; Stoller, R. E., Kumar, A. S., Gelles, D. S., Eds.; American Society for Testing and Materials: Philadelphia, PA, 1992; ASTM STP 1125, pp 928-941.

33. Norgett, M. J.; Robinson, M. T.; Torrens, I.M. Nucl. Eng. Des. 1975, 33, 50-54.

34. Bacon, D. J.; Osetsky, Yu. N.; Stoller, R. E.; Voskoboinikov, R. E. J. Nucl. Mater. 2003, 323, 152-162.

35. Calder, A. F.; Bacon, D. J.; Barashev, A. V.; Osetsky, Yu. N. On the origin of large interstitial clusters in displacement cascades. Philos. Mag. 2010, 90, 863-884.

36. Osetsky, Yu. N.; Bacon, D. J.; Serra, A.; Singh, B. N.; Golubov, S. I. One-dimensional atomic transport by clusters of self-interstitial atoms in iron and copper. Philos. Mag. 2003, Volume 83, No. 1, pages: 61-91.

37. Osetsky, Yu. N.; Bacon, D. J.; Serra, A. Philos. Mag. Lett. 1999, $79,272-282$.

38. Greenwood, G. W.; Foreman, A. J. E.; Rimmer, D. E. The role of vacancies and dislocations in the nucleation and growth of gas bubbles in irradiated fissile material. J. Nucl. Mater. 1959, 1, 305324.

39. Gosele, U. Prog. React. Kinet. 1984, 13, 63-161.
40. Brailsford, A. D.; Bullough, R.; Hayns, M. R. J. Nucl. Mater. 1976, 60, 246-256.

41. Brailsford, A. D.; Bullough, J. Nucl. Mater. 1976, 60, 257-278.

42. Brailsford, A. D.; Bullough, R. Philos. Trans. R. Soc. Lond. A 1981, 302, 87-137.

43. Stoller, R. E.; Golubov, S. I.; Becquart, C. S.; Domain, C. J. Nucl. Mater. 2008, 382, 87-137.

44. Hayns, M.R. The nucleation and early growth of interstitial dislocation loops in irradiated materials. J. Nucl. Mater. 1975, 56, 267-274.

45. Feder, J.; Russell, K. C.; Lothe, J.; Pound, G.M. Adv. Phys. 1966, $15,111-178$.

46. Wagner, R.; Kampmann, R. Materials Science and Technology, A Comprehensive Treatment; Cahn, R. W., Haasen, P., Kramer, E. J., Eds.; VCH; Weinheim, 1991; Vol. 10 B, Part II, pp 213-302.

47. Kiritani, M. J. Phys. Soc. Jpn. 1973, 56, 95-107.

48. Goodrich, F. G. Proc. R. soc. Lond. A 1964, 277, 155-166.

49. Goodrich, F. G. Proc. R. Soc. Lond. A 1964, 277, 167-182.

50. Bondarenko, A. I.; Konobeev, Yu, V. Phys. Status Solidi A 1976, 34, 195-205.

51. Ghoniem, N. M.; Sharafat, S. J. Nucl. Mater. 1980, 92, 121-135.

52. Stoller, R. E.; Robert Odette, G. $13^{\text {th }}$ International Symposium on Radiation Induced Changes in Microstructure; Garner, F. A., Packan, N. H., Kumar, A. S., Eds.; ASTM: Philadelphia, P/a, 1987; ASTM STP 955, Part I, pp 371-392.

53. Hardouin, Duparc, A; Moingeon, C.; Smetniansky-de-Grande, N.; Barbu, A. J. Nucl. Mater. 2002, 302, 143-155.

54. Wehner, M. F.; Wolfer, W. G. Vacancy cluster evolution in metals under irradiation. Philos. Mag. A 1985, Volume 52, 189-205.

55. Ghoniem, N. M. Phys. Rev. B 1989, 39, 11810-11819.

56. Surh, M. P.; Sturgeon, J. B.; Wolfer, W. G. J. Nucl. Mater. 2004, $325,44-52$.

57. Brailsford, A. D.; Bullough, R. The Rate Theory of Swelling Due to Void Growth in Irradiated Materials. J. Nucl. Mater. 1972, 44, 121-135.

58. Wiedersich, H. Radiat. Eff. 1972, 12, 11-125.

59. Integrity of reactor pressure vessels in nuclear power plants: assessment of irradiation embrittlement effects in reactor pressure vessel steels. - Vienna: International Atomic Energy Agency, 2009. p.; $29 \mathrm{~cm}$. - (IAEA nuclear energy series, ISSN 1995-7807 ; no. NP-T-3.11 STI/PUB/1382 ISBN 978-92-0-101709-3

60. Stoller, Roger. The Effect of Neutron Flux on Radiation-Induced Embrittlement in Reactor Pressure Vessel Steels. Journal of ASTM International. April 2004, Vol. 1, No. 4. Paper ID JAI11355.

61. Lee, Gyeong-Geun; Lee, Yong-Bok; Kim, Min-Chul; Kwon, Junhyun. Comparison of Rate Theory Based Modeling Calculations with the Surveillance Test Results of Korean Light Water Reactors. Transactions of the Korean Nuclear Society Autumn Meeting. Gyeongju, Korea, October 25-26, 2012.

62. S.J. Zinkle, P.J. Maziasz, R.E. Stoller. Dose dependence of the microstructural evolution in neutron irradiated austenitic stainless steel. Journal of Nuclear Materials. Volume 206, Issues 2-3, 2 November 1993, Pages 266-286.

63. Kwon, J; Motta, AT. Effect of radiation damage on BWR coreshroud cracking. Symposium on Microstructural Processes in Irradiated Materials, at the 1998 Fall MRS Meeting. Boston, MA. November 30-December 2, 1998. Volume 540, Pages 483-488. Published: 1999.

64. Kwon, Junhyun; Kim, Joo-Hag; Kwon, Sang Chul; Hong, JunHwa. Modeling of Radiation Hardening Due to Point Defect Clusters in Stainless Steels. Proceedings of the Korean Autumn Meeting. Yongpyong, Korea, October 2002.

65. Kwon, Junhyun; Kwon, Sang Chul; Hong, Jun-Hwa. Prediction of radiation hardening in reactor pressure vessel steel based on a theoretical model. Annals of Nuclear Energy. 10/2003; 30(15):1549-1559.

66. Odette, G.R.; Yamamoto, T.; Klingensmith, D. On the effect of dose rate on irradiation hardening of RPV steels. Philosophical Magazine. Vol. 85, Nos. 4-7, 01 February—01 March 2005, $779-797$.

67. Watanabe, Y.; Morishita, K.; Yamamoto, Y.; Hamaguchi, D.; Tanigawa, H. Displacement damage rate dependence of defect cluster formation in $\alpha$-Fe during irradiation. Nuclear Instruments and Methods in Physics Research B. Volume 303, p. 100-103, 2013.

68. Bergner, F.; Ulbricht, A.; Hein, H.; Kammel, M. Flux dependence of cluster formation in neutron-irradiated weld material. Journal of Physics: Condensed Matter. Vol. 20, No. 20. 24 September, 2007. 
69. Ebihara, Ken-ichi. Numerical Simulation of Irradiation-Induced Grain Boundary Phosphorous Segregation in Reactor Pressure Vessel Steels using Rate Theory Model with First Principles Calculations. Proceedings of the ASME Pressure Vessels and Piping Conference- 2009. (2010) pages: 395-402,

70. Birkenheuer, U.; Bergner, F.; Ulbricht, A.; Gokhman, A.; Almazouzi, A. Application of Rate Theory Modeling to Cluster Evolution in Binary $\mathrm{Fe}-\mathrm{Cu}$ Alloys. https://www.hzdr.de/publications/PublDoc-2473pdf. 24 February 2015.

71. Straalsund, J.L. The effect of cluster formation on the 'temperature shift' for accelerator simulation of neutron irradiation. Journal of Nuclear Materials. Volume 51, Issue 3, p. 302-308, 1974.

72. Al-Motasem, A.T.; Bergner, F.; Bonny, G.; Posselt, M.; Talati, M. Investigations of Structure, Energetics, Thermodynamics of Copper Vacancy Clusters in bcc-Fe. Accessed: 27 June 2015. http://www.csvts.cz/cns/jb/doc/papers/ENYGF2011/05 13 Talati extabs.pdf.

73. Watanabe, Y.; Morishita, K.; Nakasuji, T.; Ando, M.; Tanigawa, H. Helium effects on microstructural change in RAFM steel under irradiation: Reaction rate theory modeling. Proceedings of the $12^{\text {th }}$ International Conference on Computer Simulation of Radiation Effects in Solids. Alacant, Spain, 8-13 June 2014.

74. W. Maschek, X. Chen, F. Delage (CEA), A. Fernandez-Carretero (ITU), D.Haas (ITU), C. Matzerath Boccaccini, A. Rineiski, P. Smith (Serco), V. Sobolev (SCK CEN), R. Thetford (Serco), J. Wallenius (KTH). Accelerator driven systems for transmutation: Fuel development, design and safety. Progress in Nuclear Energy. 50 (2008) 333-340.

75. Runevall, Odd; Sandberg, Nils. Helium induced void and bubble formation in $\mathrm{MgO}$. Computational Materials Science. (2012) 60:53-58.

76. A. van Veen, H. Schut, A.V. Fedorov, F. Labohm, E.A.C. Neeft, R.J.M. Konings, $11^{\text {th }}$ International Conference on Ion Beam Modification of Materials, Aug.-Sept., 1998, Amsterdam, Nuclear Instr. And Meth. In Phys. Res. Sect. B (148) 1-4 (1999) 768-772.

77. R. W. Grimes. Solution of $\mathrm{MgO}, \mathrm{CaO}$, and $\mathrm{TiO} 2$ in alpha-A1203. Journal of the American Ceramic Society, 77(2), 1994, pp. 378384.

78. Busker, G., van Huis, M. A., Grimes, R. W. \& van Veen, A. Predicted vacancy cluster structures in $\mathrm{MgO}$ and their interaction with helium. (2000) Nucl. Instrum. Method Phys. Res. B 171, 528 536. (doi:10.1016/S0168-583X(00)00324-4)

79. $\downarrow$ Grimes R. W., Catlow C. R. A. Modeling localized defects in ionic materials using Mott-Littleton and embedded quantum cluster methodology. (1990) J. Am. Ceram. Soc 7332513256 doi:10.1111/j.1151-2916.1990.tb06446.x (doi:10.1111/j.11512916.1990.tb06446.x).

80. G. D. Tolstolutskaya, V. V. Ruzhyskyi, V.N. Voyevodin, I.E. Kopanets, S.A. Karpov, A.V. Nikitin. The role of radiation damage on retention and temperature intervals of helium and hydrogen detrapping in structural materials. Fifteenth International Conference on Fusion Reactor Materials. Volume 442, Issues 1-3, Supplement 1, November 2013, Pages S710$\mathrm{S} 714$ 